\title{
Tamaño corporal, tolerancia ecológica y potencial de bioindicación de la calidad del agua de Anacroneuria spp. (Plecoptera: Perlidae) en América del Sur
}

\author{
Sylvie Tomanova ${ }^{1,2} \&$ Pablo A. Tedesco ${ }^{2}$ \\ 1 Laboratorio de Biología de las Aguas Corrientes, Departamento de Zoología y Ecología, Universidad Masaryk, \\ Kotlárská 2, Brno, 61137, República Checa; sylvatom@seznam.cz \\ 2 UR 131 Instituto de Investigación para el Desarollo (IRD), ULRA UMSS Cochabamba, Bolivia.
}

Recibido 16-VIII-2005. Corregido 21-VI-2006. Aceptado 15-XI-2006.

\begin{abstract}
Body size, ecological tolerance and potential for water quality bioindication in the genus Anacroneuria (Plecoptera: Perlidae) from South America. Knowledge about the biology and ecology of neotropical aquatic taxa is crucial to establish general ecological rules and water protection systems. Based mainly on published data, the present work shows the following biological and ecological characteristics of Anacroneuria species (Klapálek 1909): a) the wide range of environmental conditions of rivers where Anacroneuria species occur, b) species number decreases along an increasing elevation gradient, c) body size increases in relation to the maximum altitude of occurrence, d) altitudinal range increases with body size, e) there is a constant relationship between male and female body size, and finally, f) larger females lay larger eggs. In temperate countries, the family Perlidae in general, and the genus Anacroneuria in particular, are viewed as excellent water quality indicators. We suggest that, considering the complexity of the group's ecology in South America, it should not be automatically considered an excellent bioindicator in the Neotropical region. Rev. Biol. Trop. 55 (1): 67-81. Epub 2007 March. 31.
\end{abstract}

Key words: Plecoptera, Anacroneuria, ecological tolerance, altitudinal range, body size, egg size, biological indexes.

Se ha despertado en los últimos años un gran interés por el uso de los macroinvertebrados como bioindicadores de la calidad de agua en América del Sur (e.g. Jacobsen 1998, Fernández et al. 2001, Marques y Barbosa 2001, Fenoglio et al. 2002, Figueroa et al. 2003). Sin embargo, su implementación es complicada por el poco conocimiento de la taxonomía de los organismos y de sus niveles de tolerancia a las condiciones ambientales. Por esta razón, se ha seguido los sistemas de medición de la calidad de agua desarollados en la zona templada (e.g. Family Biotic index, BWMP, ASPT). Estos sistemas calculan índices biológicos basados en valores de tolerancia de las familias frente a una contaminación.
En realidad, el valor de tolerancia de cada familia representa un valor promedio de los valores de tolerancia de los géneros o especies de la familia. Lamentablemente, estos valores han sido determinados para los taxones de la zona templada, por lo cual los sistemas están adaptados sólo para esta zona. Los valores de tolerancia de los organismos frente a una contaminación tendrían que ser recalificados antes de ser usados en otros países, si no, la aplicación de estos sistemas puede resultar defectuosa (Pavluk et al. 2000). Sin embargo, para poder recalificar la tolerancia de los organismos y luego evaluar la perturbación de los ecosistemas, es necesario examinar primero las relaciones ecológicas y funcionales entre 
los organismos y su medio ambiente (Bonada et al. 2004).

El género Anacroneuria (Klapálek 1909) es uno de los más importantes en la fauna de los plecópteros de la zona neotropical. Su área de distribución cubre desde América del Sur y Central (Jewett 1958, Romero 2001, Bispo et al. 2002a, b) hasta Arizona en los Estados Unidos (Stark 2001b). El gran interés que despertó este género durante los últimos años (Stark 1994, 1995, 1998, 1999, 2000, Stark y Sivec 1998, Froehlich 2000, 2001a, Maldonado et al. 2002, Stark y Zúñiga 2003, Orce 2003) condujo a la descripción de alrededor de 200 especies y se supone que el número total de especies en el neotrópico excede las 300 (Stark 2001b). Sin embargo, la variabilidad de las características biológicas y ecológicas de las especies de este género no ha sido estudiada.

El presente trabajo recoge y analiza información publicada en las descripciones de especies del género Anacroneuria, complementadas con datos provenientes de ríos de Bolivia. Basándonos en esa información, presentamos algunas variables biológicas y ecológicas de las especies en relación con las preguntas siguientes: 1) ¿Se puede usar el valor de tolerancia ecológica de la familia Perlidae (a menudo representada por el género Anacroneuria) de la zona templada para la zona neotropical? 2) ¿Cómo varía el número de especies con la altitud máxima de presencia? 3) ¿Cómo varía el tamaño corporal con la altitud máxima de presencia? 4) Hay alguna relación entre el tamaño corporal y la amplitud del ámbito altitudinal?

\section{MATERIALES Y MÉTODOS}

Para evaluar la tolerancia ecológica del género Anacroneuria en la zona neotropical, resumimos los valores máximos y mínimos de algunas características generales y físicoquímicas de los ríos en los cuales se notó la presencia de este género. Estos valores provienen de trabajos anteriores en la zona neotropical (Stark 2001a, Baptista et al. 2001a, b, Bobot y Hamada 2002, y Moya et al. 2003) y de nuestros propios datos de campo provenientes de los ríos de Bolivia. Para establecer otras características biológicas y ecológicas del género Anacroneuria, se buscaron datos primarios en publicaciones anteriores dedicadas a descripciones de nuevas especies para América del Sur (ver el Apéndice). Nos interesamos especialmente en la altitud máxima y mínima de presencia de las especies, el tamaño máximo del macho ( $\sigma^{x}$ ) y de la hembra ( $Q$ ) ( $\mathrm{mm}$ ) en estado adulto y en la medida del tamaño del huevo, que se determinó según la suma de su largo y ancho (mm). Esta información no está disponible para todas las especies descritas (ver el Apéndice). Por ejemplo, se conoce mejor el tamaño $\sigma^{\top}$ que el tamaño $\bigcirc$. Primero se estableció la variabilidad del número de especies sobre la gradiente de altitud máxima. Luego se observó cómo varía el tamaño $\sigma^{\top}$ de cada una de las especies con la altitud máxima donde se encuentran. Se estimó el ámbito altitudinal para cada especie como la diferencia de altitud máxima y mínima de su presencia, y se lo relacionó con el tamaño $O^{\top}$ de las especies. Además, se analizó la relación entre el tamaño $\delta^{\pi}$ y $Q$ de las especies, y la relación entre el tamaño $q$ y el tamaño de los huevos. Al no tener una distribución normal, estas relaciones fueron analizadas estadísticamente con correlaciones no paramétricas de rangos de Spearman.

\section{RESULTADOS}

Las especies del género Anacroneuria se encuentran en ríos desde 10 a $3500 \mathrm{~m}$ de altura (Cuadro 1), del orden 1 a 6 con una anchura del río desde 0.5 a $97 \mathrm{~m}$. Las nínfas prefieren las partes rápidas del río (velocidad hasta $2.99 \mathrm{~ms}^{-1}$ ) pero se pueden encontrar también en lugares sin corriente. Son organismos euritermos y soportan temperaturas desde 15 hasta $33{ }^{\circ} \mathrm{C}$. Las especies de Anacroneuria se encuentran en los ríos bajo- y sobre-saturados en oxígeno (5.2-12 $\mathrm{mgl}^{-1}$ o $64-139 \%$ de saturación), con un $\mathrm{pH}$ desde ácido hasta básico (4-8.76), y de conductividad desde 11 a 795 $\mu \mathrm{Scm}^{-1}$. Todas estas características muestran 
CUADRO 1

Valores mínimos y máximos de los parámetros físicos de los ríos neotropicales con larvas de Anacroneuria presentes

Parámetros

$\begin{array}{cc}\text { Min } & \text { Max } \\ 10(4) & 3500(1) \\ 1 & 6(2) \\ 0.5(3) & 97(5) \\ 0.14 & 0.82 \\ 0 & 2.99(4) \\ 14.67(4) & 33(5) \\ 5.2(5) & 12 \\ 64(5) & 139 \\ 4(3) & 8.76 \\ 11 & 795\end{array}$

Según (1) Stark (2001a), (2) Baptista et al. (2001a), (3) Bobot y Hamada (2002), (4) Baptista et al. (2001b), (5) Moya et al. (2003) y nuestros datos no publicados de Bolivia.

que las nínfas del género Anacroneuria difieren ampliamente en sus valores de tolerancia frente a las condiciones ambientales.

A partir de la altitud máxima de presencia de 117 especies descritas hasta ahora, el número de especies disminuye significativamente con la altitud ( $\rho=-0.70, p=0.003$; Fig. 1). Esta tendencia parece ser generada principalmente por el escaso número de especies con altitudes máximas superiores a los $2400 \mathrm{~m}$. El tamaño $\mathrm{O}^{\top}$ de las especies aumenta significativamente con la altitud máxima de presencia $(\rho=0.79$, $\mathrm{p}<0.001, \mathrm{n}=101$; Fig. 2A). Al mismo tiempo, las especies más grandes son capaces de ocupar un ámbito altitudinal más grande $(\rho=0.46$, $\mathrm{p}=0.002, \mathrm{n}=45$; Fig. 2B). Se observó en casi todos los casos que el macho es más pequeño que la hembra, y el tamaño $\sigma^{\top}$ se relacionó muy claramente con el tamaño $\bigcirc(\rho=0.91, p<0.001$, $\mathrm{n}=69$; Fig. 2C). Se encontró también una relación significativa y positiva entre el tamaño $O$ de las especies y el tamaño del huevo $(\rho=0.38$, $\mathrm{p}=0.02, \mathrm{n}=30$; Fig. 2D).

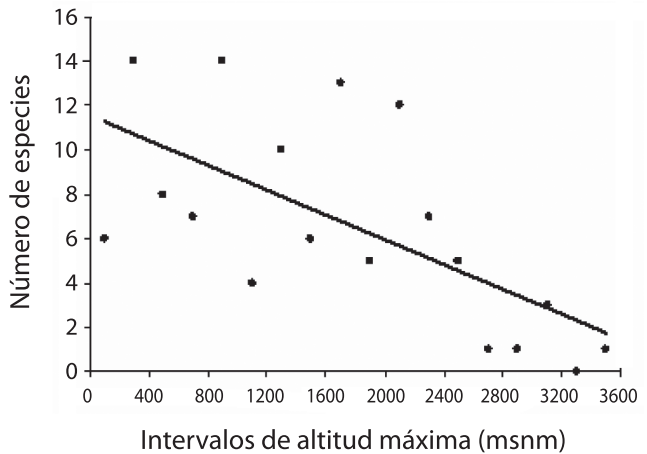

Fig. 1. Número de especies del género Anacroneuria por intervalos de altitud máxima ( $\mathrm{msnm})$.

\section{DISCUSIÓN}

Potencial de bioindicación: en la zona templada, la familia Perlidae es indicadora de buena calidad del agua porque es intolerante a la deficiencia en oxígeno. Prefiere las aguas oligotróficas y oligo- o $\beta$-mesosapróbicas (Tachet et al. 2000), las cuales son generalmente saturadas en oxígeno (Friedrich et al. 1992). Al contrario, en la zona neotropical, como indican los datos antes analizados, la familia Perlidae puede encontrarse en condiciones de bajo-saturación en oxígeno. De esta manera, se puede concluir que algunas especies de la familia Perlidae de la zona neotropical pueden tolerar un cierto grado de la contaminación orgánica, relacionada a la deficiencia en oxígeno. Bispo et al. (2002b) confirman esta conclución al encontrar nínfas de Anacroneuria en ríos caracterizados por un grado de contaminación antropogénica de nulo hasta alto. Aunque los datos presentados aquí no sean exhaustivos, podemos concluir que ni la familia Perlidae, y aún menos el género Anacroneuria, pueden figurar automáticamente como indicadores de buena calidad del agua en la zona neotropical. Los valores de tolerancia (de Perlidae) frente a una contaminación en los sistemas Family biotic index, BMWP y ASPT tendrían que ser recalificados antes de ser usados en la zona neotropical. 

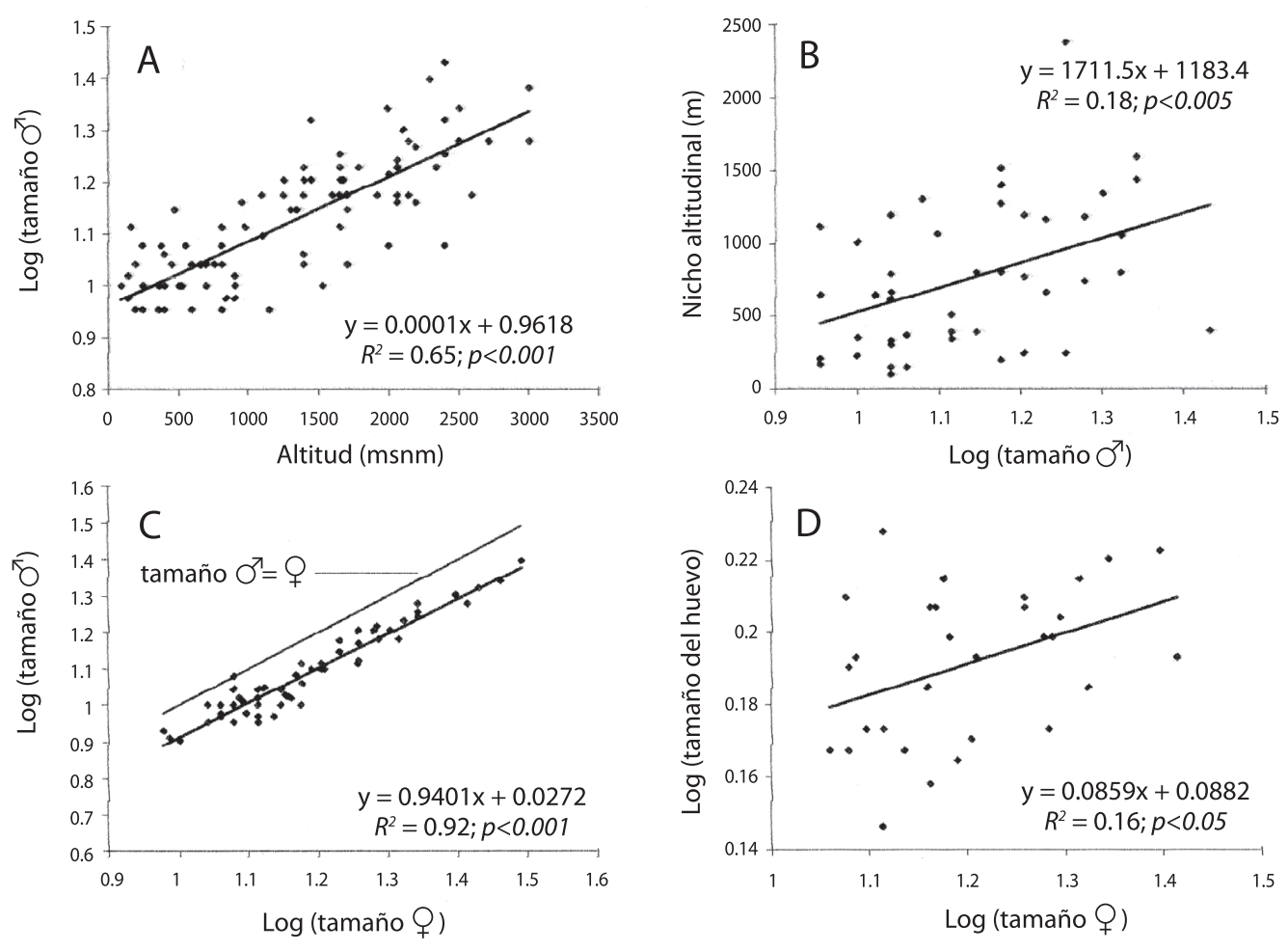

Fig. 2. Algunas relaciones ecológicas de las especies de Anacroneuria: a) variación del tamaño $\sigma^{\top}$ con la altitud máxima $(\mathrm{msnm})$ de la presencia de las especies $(n=101$ especies); b) estimación del ámbito altitudinal en relación con el tamaño $\sigma^{\top}(n=45)$; c) relación entre tamaño $\sigma^{\top}$ y tamaño $\subsetneq(n=69)$; y d) tamaño del huevo en relación conl tamaño $\subsetneq(n=30)$. Regresiones lineales simples muestran las tendencias generales.

Según la hipótesis de Frutiger (1996), los taxones que tienen una distribución geográfica extensa, deben ser capaces de sobrevivir una gran variedad de condiciones ambientales a las cuales deben estar adaptados (Townsend y Hildrew 1994). Probablemente, distintas condiciones ambientales estimularon la divergencia de estos taxones (Species SpeciationGaston 2003) y cada especie generada podría caracterizarse por diferentes exigencias ecológicas. Bonada et al. (2004) mostraron que, en el caso de los tricópteros de la zona templada, las tolerancias ecológicas de las especies dentro de una familia taxonómicamente diversa son mucho más diferentes que en una familia poco diversa. En este caso, el uso de la tolerancia promedio por familia, como ocure por exemplo con el sistema BMWP, puede causar resultados falsos. Según Fernández y
Domínguez (2001), muchos géneros de invertebrados acuáticos tienen amplias distribuciones por toda América del Sur. Aunque el conocimiento taxonómico sea aún escaso, es de notar que en la zona tropical las familias y los géneros son más diversos que en la zona templada (Jacobsen et al. 1997). Siguiendo el ejemplo de Anacroneuria, podriamos suponer que otras familias/géneros con amplia distribución geográfica (e.g. Helicopsyche, Hydroptila-Trichoptera; Thraulodes, Baetodes, Tricorythodes-Ephemeroptera; etc.) manifestan, de la misma manera, grandes diferencias de tolerancia ecológica entre las especies. De esta manera, se puede perder el significado a nivel de familias/géneros para la bioindicación porque al usar el valor promedio por familia/ género se pierde la verdadera información ecológica. De confirmarse este hecho, los sistemas 
de calidad de agua que operan con valores promedio a nivel de famila o género podrían ser inefectivos en la zona neotropical.

\section{Características biológicas y ecológicas:} la disminución del número de especies en función de la altitud máxima de presencia concuerda con relaciones negativas entre altitud y riqueza encontradas anteriormente (e.g. Jones et al. 2003, Vinson y Hawkins 2003), aunque relaciones positivas también han sido observadas (Romero-Alcaraz y Avila 2000, Sanders 2002). Teniendo en cuenta que las especies de gran altitud máxima también se pueden encontrar a más baja altitud, pero no vice-versa, la tendencia observada debería ser más marcada en caso de evaluar la riqueza específica de localidades según una gradiente de altura. De esta manera, la riqueza específica podría reflejar la disminución con la altura de varios componentes del habitat tales como la productividad, la heterogeneidad espacial o la superficie disponible (Townsend et al. 2002), necesarios para la coexistencia de las especies.

Los rasgos biológicos de las especies resultan de adaptaciones a las differentes presiones ambientales, bióticas y abióticas (Townsend y Hildrew 1994). El tamaño corporal es una característica muy importante relacionada a varios rasgos ecológicos, biológicos y fisiológicos (Peters 1983). Nuestros resultados muestran un aumento del tamaño corporal de los machos de diferentes especies del género Anacroneuria con la altitud. Las relaciones lineales entre el tamaño $O^{\top}$ y $O$, y entre el tamaño $q$ y del huevo indican que también las hembras y los huevos siguen el mismo patrón. El tamaño de los adultos debe depender directamente del tamaño de las larvas porque los plecópteros son insectos hemimetábolos. Tomando en cuenta el tiempo del estado adulto de los plecópteros, que puede durar desde pocos días hasta varias semanas, este es menos importante que el estado de las larvas, que viven desde varios meses hasta varios años (Romero 2001). Para tratar de explicar la variabilidad del tamaño con la altitud entre las especies del género Anacroneuria, nos basamos en el hecho que el tamaño del adulto se relaciona con el tamaño del huevo y de la larva (nuestros resultados, Lillehammer et al. 1989 en Gillooly y Dobson 2000, Wilhelm y Schindler 2000), y que el tamaño resultante debe presentar una ventaja durante la mayor parte del ciclo de vida (huevo y larva).

Los ríos de baja altitud tienen una cantidad variable de oxígeno, desde sobre- hasta bajosaturación (Moya et al. 2003). En condiciones de bajo-saturación, el tamaño pequeño del huevo facilita la difusión del oxígeno y así asegura un mejor desarrollo del mismo. La cantidad de oxígeno en la agua puede entonces influir sobre la supervivencia de los huevos en función del tamaño de estos y asi determinar la presencia o la ausencia de las especies. Por otro lado, el tamaño del huevo se relaciona positívamente con el tamaño natal (Lillehammer et al. 1989 en Gillooly y Dobson 2000, Wilhelm y Schindler 2000). Comparando el desarollo de los individuos de Gammarus lacustris (Crustacea) provenientes de huevos de varios tamaños en aguas frías, Wilhelm y Schindler (2000) demostraron que después del nacimiento, la mortandad de los grandes individuos es más pequeña que la de los individuos de menor tamaño. Así la temperatura puede figurar como otro factor límite influenciando el tamaño de las especies.

Las características morfológicas de las larvas pueden ser dirigidas tanto por factores abióticos como bióticos. Existen varias hipótesis que pueden explicar el aumento del tamaño corporal con la altitud. Krasnov et al. (1996) proponen que los habitats más productivos (ríos tropicales de baja altitud en nuestro caso) pueden producir más individuos y que una elevada compentencia intra-específica por los alimentos induce una disminución del tamaño corporal. El género Anacroneuria debería entonces presentar mayor abundancia de individuos pequeños en los ríos de baja altitud. Sin embargo, nuestras observaciones de terreno son contrarias a esta predicción (abundacia media durante la época seca de $41 \mathrm{ind} / \mathrm{m}^{2}$ a $1807 \mathrm{~m}$ de altura y de $28 \mathrm{ind} / \mathrm{m}^{2}$ a $399 \mathrm{~m}$; Tomanova, no publ.). Por otro lado, la varibilidad del tamaño corporal puede ser explicada por la 
depredación y la composición del substrato. En los ríos de montaña el substrato es generalmente más grande y los individuos de gran tamaño pueden refugiarse dentro el substrato y así escapar a la depredación por parte de los peces. Además, la abundancia de peces disminuye en los ríos de altura (Flecker y Feifarek 1994), con lo cual diminuye la presión de la depredación. En el substrato menos grueso de los ríos de baja altitud los individuos de gran tamaño tienen menos posibilidades de refugiarse y presentan un mayor riesgo de depredación. En consequencia, el tamaño resultante de las especies de Anacroneuria puede ser determinado por la selección por depredación y por la disponibilidad de refugios. Esta hipótesis se apoya también en los resultados de Peckarsky et al. (2001) quienes muestran una disminución del tamaño corporal del género Baetis (Ephemeroptera) como reacción al riesgo de predación en los arroyos poblados por peces.

Las informaciones biológicas y ecológicas sobre las especies neotropicales son muy escasas. Se necesita una gran cantidad de estudios de base y/o de síntesis de este tipo para poder comprender mejor el funcionamiento de los ecosistemas naturales de la zona neotropical. Esta es una condición necesaria para establecer las reglas ecológicas generales para la conservación de la riqueza natural de este continente y para un uso adecuado de los índices bióticos que sirven para el manejo de dicha riqueza.

\section{AGRADECIMIENTOS}

Gracias a Núria Bonada y dos revisores anónimos por sus sugerencias, que ayudaron a mejorar el artículo. Este trabajo es el resultado de una colaboración científica entre el IRD (Institut de Recherche por le Développement) de Francia, la ULRA (Unidad de Limnología y Recursos Acuáticos) de la UMSS de Bolivia y el Departamento de Biología de las Aguas Corrientes de la Universidad Masaryk de la República Checa. Este estudio ha sido realizado dentro del marco del plan de investigación de la Universidad Masaryk (Ministerio de Educación de la República Checa, MSM 0021622416).

\section{RESUMEN}

El conocimiento sobre la biología y la ecología de los organismos acuáticos neotropicales es de gran importancia para evaluar reglas ecológicas generales y para establecer un sistema de protección de los recursos acuáticos. Basado principalmente sobre datos recoleccióndos en publicaciones anteriores, el presente trabajo muestra las siguientes características biológicas y ecológicas de las especies del género Anacroneuria (Klapálek 1909; Perlidae: Plecoptera): a) la gran amplitud de las condiciones ambientales de los ríos poblados por el género Anacroneuria, b) la disminución del número de especies sobre una gradiente de altitud, c) el aumento del tamaño corporal con la altitud máxima, d) el aumento del ámbito altitudinal con el tamaño corporal, e) la relación constante entre el tamaño del macho y de la hembra, y finalmente, f) el aumento del tamaño de los huevos con el tamaño de la hembra. Sugerimos que la familia Perlidae y el género Anacroneuria no debrían ser automaticamente considerados como excelentes indicadores de la calidad del agua en la zona neotropical.

Palabras clave: Plecoptera, Anacroneuria, tolerancia ecológica, ámbito altitudinal, tamaño corporal, tamaño de los huevos, indices biológicos.

\section{REFERENCIAS}

Baptista, D.F., D.F. Buss, L.F. M. Dorville \& J.L. Nessimian. 2001a. Diversity and habitat preference of aquatic insects along the longitudinal gradient of the Macae river basin, Rio de Janeiro, Brazil. Rev. Brasil. Biol. 61: 249-258.

Baptista, D.F., L.F.M. Dorville, D.F. Buss \& J.L. Nessimian. 2001b. Spatial and temporal organization of aquatic insects assemblages in the longitudinal gradient of a tropical river. Rev. Brasil. Biol. 61: 295-304.

Bispo, P.C., C.G. Froehlich \& L.G. Oliveira. 2002a. Stonefly (Plecoptera) fauna in a mountainous area of Central Brazil: composition and adult phenology. Rev. Brasil. Zool. 19: 317-323.

Bispo, P.C., C.G. Froehlich \& L.G. Oliveira. 2002b. Stonefly (Plecoptera) fauna of streams in a mountainous area of Central Brazil: abiotic factors and nymph density. Rev. Brasil. Zool. 19: 325-334.

Bobot, T.E. \& N. Hamada. 2002. Plecoptera genera of two streams in Central Amazonia, Brazil. Entomotropica 17: 299-301. 
Bonada, N., C. Zamora-Muñoz, M. Rieradevall \& N. Prat. 2004. Ecological profiles of caddisfly larvae in Mediterranean streams: implications for bioassessment methods. Environ. Pollut. 132: 509-521.

Fenoglio, S., G. Badino \& F. Bona. 2002. Benthic macroinvertebrate communities as indicators of river environment quality: an experience in Nicaragua. Rev. Biol. Trop. 50: 1125-1131.

Fernández, H.R. \& E. Domínguez. 2001. Guía para la determinación de los artrópodos bentónicos sudamericanos. Universidad Nacional de Tucumán, Tucumán, Argentina. 282 p.

Fernández, H.R., F. Romero, M. Peralta \& L. Grosso. 2001. La diversidad del zoobentos en ríos de montaña del noroeste de Argentina: comparación entre seis ríos. Austral Ecol. 11: 9-16.

Figueroa, R., V.C., E. Araya \& O. Parra. 2003. Macroinvertebrados bentónicos como indicadores de calidad de agua de ríos del sur de Chile. Rev. Chil. Hist. Nat. 76: 275-285.

Flecker, A.S. \& B. Feifarek. 1994. Disturbance and the temporal variability of invertebrate assemblages in two Andean streams. Fresh. Biol. 31: 131-142.

Friedrich, G., D. Chapman \& A. Beim. 1992. The use of biological material, p. 171-238. In D. Chapman (ed.). Water Quality Assessments - A Guide to Use of Biota, Sediments and Water in Environmental Monitoring. Chapman and Hall, Londres, Inglaterra.

Froehlich, C.G. 2000. Biological observations on Tupiperla (Plecoptera: Gripopterygidae). Rev. Brasil. Biol. 60: 447-450.

Froehlich, C.G. 2002. Anacroneuria mainly from southern Brazil and northeastern Argentina (Plecoptera: Perlidae). P. Biol. Soc. Wash. 115: 75-107.

Froehlich, C.G. 2003. Stoneflies (Plecoptera: Perlidae) from the Brazilian Amazonia with the description of three new species and a key to Macrogynoplax. Stud. Neotrop. Fauna E. 38: 129-134.

Frutiger, A. 1996. Embryogenesis of Dinocras cephalotes, Perla grandis and P. marginata (Plecoptera: Perlidae) in different temperature regimes. Fresh. Biol. 36: 497-508.

Gaston, K.J. 2003. The structure and dynamics of geographic ranges. Oxford University, Oxford, Inglaterra. $266 \mathrm{p}$.

Gillooly, J.F. \& S.I. Dodson. 2000. The relationship of egg size and incubation temperature to embry- onic development time in univoltine and multivoltine aquatic insects. Fresh. Biol. 44: 595-604.

Jacobsen, D. 1998. The effect of organic pollution on the macroinvertebrate fauna of Ecuadorian highland streams. Arch. Hydrobiol. 143: 179-195.

Jacobsen, D., R. Schultz \& A. Encalada. 1997. Structure and diversity of stream invertebrate assemblages: the influence of temperature with altitude and latitude. Fresh. Biol. 38: 247-261.

Jewett, S.G. 1958. Stoneflies of the genus Anacroneuria from Mexico and Central America (Plecoptera). Am. Midl. Nat. 60: 159-175.

Jones, J.L., W. Li \& S.C. Maberly. 2003. Area, altitude and aquatic plant diversity. Ecography 26: 411-420.

Krasnov, B., D. Ward \& G. Shenbrot. 1996. Body size and leg length variation in several species of darkling beetles (Coleoptera: Tenebrionidae) along a rainfall and altitudinal gradient in the Negev Desert (Israel). J. Arid Environ. 34: 477-489.

Lillehammer, A., J.E. Brittain, S.J. Saltveit \& P.S. Nielsen. 1989. Egg development, nymphal growth and life cycle strategies in Plecoptera. Holarctic Ecol. 12: 173-186.

Maldonado, V., B.P. Stark \& C. Cressa. 2002. Descriptions and records of Anacroneuria from Venezuela (Plecoptera: Perlidae). Aquat. Insect. 24: 219-236.

Marques, M.M. \& F. Barbosa. 2001. Biological quality of waters from an impacted tropical watershed (middle Rio Doce basin, southeast Brazil), using benthic macroinvertebrate communities as an indicator. Hydrobiologia 457: 69-76.

Moya, N., E. Goitia \& M. Siles. 2003. Tipología de ríos de la región del Piedemonte Andino en Cochabamba. Rev. Bol. Ecol. 13: 95-115.

Orce, M. 2003. A new species of Anacroneuria from Nortwestern Argentina (Plecoptera: Perlidae), p. 225-228. In E. Gaino (ed.). Research Update on Ephemeroptera and Plecoptera. University of Perugia, Perugia, Italia

Pavluk, T.I., A. Bij de Vaate \& H.A. Leslie. 2000. Development of an index of trophic completeness for benthic macroinvertebrate communities in flowing waters. Hydrobiologia 427: 135-141.

Peckarsky, B.L., B.W. Taylor, A.R. McIntosh, M.A. McPeek \& D.A. Lytle. 2001. Variation in mayfly size at metamorphosis as a developmental response to risk of predation. Ecology 82: 740-757. 
Peters, R.H. 1983. The ecological implications of body size. Cambridge University, Inglaterra. 345 p.

Ribeiro-Ferreira, A.C. \& C.G. Froehlich. 2001. Anacroneuria Klapálek, 1909 from Amazonas state, north Brazil (Plecoptera, Perlidae, Acroneuriinae). Aquat. Insect 23: 187-192.

Romero, V.F. 2001. Plecoptera, p. 93-109. In H.R. Fernández \& E. Domínguez (eds.). Guía para la determinación de los artrópodos bentónicos sudamericanos. Universidad Nacional de Tucumán, Tucumán, Argentina.

Romero-Alcaraz, E. \& J.M. Avila. 2000. Effect of elevation and type of habitat on the abundance and diversity of Scarabaeoid dung beetle (Scarabaeoidea) assemblages in a Mediterranean area from Southern Iberian Peninsula. Zool. Stud. 39: 351-359.

Sanders, N.J. 2002. Elevational gradients in ant species richness: area, geometry, and Rapoport's rule. Ecography 25: 25-32.

Stark, B.P. 1994. Anacroneuria of Trinidad and Tobago (Plecoptera: Perlidae). Aquat. Insect. 16: 171-175.

Stark, B.P. 1995. New species and records of Anacroneuria (Klapálek) from Venezuela. Spixiana 18: 211-249.

Stark, B.P. 1998. The Anacroneuria of Costa Rica and Panama (Insecta: Plecoptera: Perlidae). P. Biol. Soc. Wash. 111: 551-603.

Stark, B.P. 1999. Anacroneuria from northeastern South America (Insecta: Plecoptera: Perlidae). P. Biol. Soc. Wash. 112: 70-93.

Stark, B.P. 2000. Notes on the Anacroneuria (Plecoptera: Perlidae) of Guyana with the description of a new species. Aquat. Insect. 22: 305-310.

Stark, B.P. 2001a. Records and descriptions of Anacroneuria from Ecuador (Plecoptera: Perlidae). Scopolia 46: 1-42.
Stark, B.P. 2001b. A synopsis of neotropical Perlidae (Plecoptera), p. 405-422. In E. Domínguez (ed.). Trends in Research in Ephemeroptera and Plecoptera. Kluwer Academic/Plenum, Nueva York, Nueva York, EEUU.

Stark, B.P. 2004. New species and records of Andean Anacroneuria (Insecta: Plecoptera: Perlidae). Spixiana 27: 67-81.

Stark, B.P. \& I. Sivec. 1998. Anacroneuria of Peru and Bolivia (Plecoptera: Perlidae). Scopolia 40: 1-64.

Stark, B.P. \& M.C. Zúñiga. 2003. The Anacroneuria guambiana complex of South America (Plecoptera: Perlidae), p. 229-237. In E. Gaino (ed.). Research Update on Ephemeroptera \& Plecoptera. Universidad de Perugia, Perugia, Italia.

Stark, B.P., M.C. Zúñiga \& I. Sivec. 2001. Descriptions of Anacroneuria spp. (Plecoptera: Perlidae) from the upper río Amazonas drainage, Colombia and Peru. Acta Entomol. Slovenica 9: 119-122.

Tachet, H., P. Richoux, M. Bournaud \& P. UsseglioPolatera. 2000. Invertébrés d'eau douce; systématique, biologie, écologie. CNRS. Paris. 588 p.

Townsend, C.R., M. Begon \& J.L. Harper. 2002. Essentials of Ecology. Blackwell Science, Massachusetts, EEUU. p. 550

Townsend, C.R. \& A.G. Hildrew. 1994. Species traits in relation to habitat templet for river systems. Fresh. Biol. 31: 265-275.

Vinson, M.R. \& C.P. Hawkins. 2003. Broad-scale geographical patterns in local stream insect genera richness. Ecography 26: 751-767.

Wilhelm, F.M. \& D.W. Schindler. 2000. Reproductive strategies of Gammarus lacustris (Crustacea: Amphipoda) along an elevation gradient. Funct. Ecol. 14: 413-422. 


\section{APÉNDICE}

\section{Resumen de los datos encontrados}

Códigos: (*) Especie no utilizada por falta de información; (arg) Argentina; (bo) Bolivia; (bra) Brazil; (col) Colombia; (cr) Costa Rica; (ecu) Ecuador; (guy) Guyana; (ho) Honduras; (mex) México; (nic) Nicaragua; (pa) Panamá; (per) Perú; (sur) Suriname; (ve) Venezuela.

\begin{tabular}{|c|c|c|c|c|c|c|c|c|}
\hline \multirow[t]{2}{*}{ Anacroneuria sp. } & \multicolumn{2}{|c|}{$\begin{array}{c}\text { Tamaño del cuerpo } \\
\text { (mm) }\end{array}$} & \multicolumn{2}{|c|}{$\begin{array}{l}\text { Huevo } \\
\text { (mm) }\end{array}$} & \multicolumn{2}{|c|}{$\begin{array}{l}\text { Altura } \\
(\mathrm{m})\end{array}$} & \multirow[t]{2}{*}{ País } & \multirow[t]{2}{*}{ Bibliografía } \\
\hline & Macho & Hembra & Ancho & Largo & Máxima & Minima & & \\
\hline achagua* & 8 & & & & & & ve & Stark 1999 \\
\hline acutipennis & 17 & 21 & & & 1650 & 1400 & $\mathrm{cr}$ & Stark 1998 \\
\hline adamsae & 14 & & & & 2150 & & pe, arg & Stark y Sivec 1998 \\
\hline alajuela* & 9 & & & & & & $\mathrm{cr}$ & Stark 1998 \\
\hline amaru & 16.5 & & & & 2060 & 2030 & bo & Stark 2004 \\
\hline amboro & 14 & & & & 2060 & & bo & Stark 2004 \\
\hline anchicaya* & & & & & 1250 & 650 & ecu, col & Stark 2001a \\
\hline angusticollis & 14 & & & & 1920 & 650 & ecu & Stark 2001a \\
\hline annulipalpis & 16 & 20 & & & 1650 & 980 & $\mathrm{cr}$ & Stark 1998 \\
\hline arawak & 9 & 10.5 & & & 500 & & sur, guy & Stark 1999; 2000 \\
\hline arcuata & 14 & & & & 1100 & & ve & Stark 1995 \\
\hline aroucana & 8 & 11 & 0.2 & 0.35 & 810 & & $\begin{array}{l}\text { Trinidad } \\
\text { Island, ve }\end{array}$ & Stark 1994; Stark 1999 \\
\hline arrazayalensis & 10 & & & & 660 & & $\arg$ & Orce 2003 \\
\hline atrinota & 9.5 & 13.5 & & & & & pe & Stark y Sivec 1998 \\
\hline auca & 9 & & & & 400 & & ecu & Stark 2001a \\
\hline aymara & 9 & & & & 900 & 550 & pe, ecu & $\begin{array}{l}\text { Stark y Sivec 1998; } \\
\text { Stark 2001a }\end{array}$ \\
\hline badilinea & & 18.7 & 0.33 & 0.27 & & & bra, arg & Froehlich 2002 \\
\hline baniva & 7.5 & 8.5 & & & & & ve & Stark 1995 \\
\hline bari & 9 & 12 & & & 1530 & 520 & ve, ecu & Stark $1995 ; 1999 ; 2001 \mathrm{a}$ \\
\hline benedettoi & 10 & 13 & & & 700 & 35 & $\mathrm{cr}$ & Stark 1998 \\
\hline bifasciata & 11.5 & 15 & 0.2 & 0.28 & 1100 & 30 & ve & Stark 1995; 1999 \\
\hline bipunctata* & & 14 & & & 250 & & pe & Stark 2004 \\
\hline blanca & 12 & 14 & 0.19 & 0.45 & 160 & & ve & Stark 1995 \\
\hline blanda* & 9 & 14 & & & & & $\mathrm{cr}$ & Stark 1998 \\
\hline brunneilata* & 12 & 17 & & & & & pe & Stark y Sivec 1998 \\
\hline bulbosa* & 15 & & & & & & pe & Stark y Sivec 1998 \\
\hline cacute & 13.5 & & & & 2200 & & ve & Maldonado et al. 2002 \\
\hline callanga & 13 & & & & 1300 & & pe & Stark y Sivec 1998 \\
\hline camposi* & 24 & & & & & & ecu & Stark 2001a \\
\hline cana & 15 & & & & 1670 & & pe & Stark y Sivec 1998 \\
\hline
\end{tabular}




\section{APÉNDICE (Continuación)}

\section{Resumen de los datos encontrados}

Códigos: (*) Especie no utilizada por falta de información; (arg) Argentina; (bo) Bolivia; (bra) Brazil; (col) Colombia; (cr) Costa Rica; (ecu) Ecuador; (guy) Guyana; (ho) Honduras; (mex) México; (nic) Nicaragua; (pa) Panamá; (per) Perú; (sur) Suriname; (ve) Venezuela.

\begin{tabular}{|c|c|c|c|c|c|c|c|c|}
\hline \multirow[t]{2}{*}{ Anacroneuria sp. } & \multicolumn{2}{|c|}{$\begin{array}{l}\text { Tamaño del cuerpo } \\
(\mathrm{mm})\end{array}$} & \multicolumn{2}{|c|}{$\begin{array}{l}\text { Huevo } \\
(\mathrm{mm})\end{array}$} & \multicolumn{2}{|c|}{$\begin{array}{l}\text { Altura } \\
(\mathrm{m})\end{array}$} & \multirow[t]{2}{*}{ País } & \multirow[t]{2}{*}{ Bibliografía } \\
\hline & Macho & Hembra & Ancho & Largo & Máxima & Minima & & \\
\hline canchi & 9 & & & & 250 & & pe & Stark y Sivec 1998 \\
\hline canelo & 13.5 & & & & 950 & & ecu & Stark 2001a \\
\hline caraca $^{*}$ & & & & & 550 & & ve, ecu & Stark 2001a \\
\hline caraca & 10 & 12 & 0.17 & 0.32 & 600 & 500 & ve & Stark 1995; 1999 \\
\hline caraja* & 11.2 & & & & & & bra & Froehlich 2002 \\
\hline carchi & 14 & & & & 1250 & & ecu & Stark 2001a \\
\hline carole* & & 11 & & & 250 & & pe & Stark 2004 \\
\hline cathia & 11.5 & 15.2 & 0.2 & 0.36 & & & bra & Froehlich 2002 \\
\hline cayapa* & 10 & & & & & & ecu & Stark 2001a \\
\hline chaima & 8 & & & & 810 & & ve & Stark 1999 \\
\hline chavin* & 10.5 & & & & & & pe & Stark y Sivec 1998 \\
\hline chimborazo & 16 & & & & 2340 & & ecu & Stark 2001a \\
\hline chipaya & 18 & 21 & & & 2500 & & bo & Stark y Sivec 1998 \\
\hline chiquita* & 8 & & & & & & ve & Stark 1995 \\
\hline chorrera & 18 & 25 & 0.21 & 0.35 & 2720 & 1530 & ve & Stark 1995; 1999 \\
\hline claudiae & 8 & & & & 360 & 150 & ve & Stark 1999 \\
\hline cochabamba & 13.5 & & & & 2060 & & bo & Stark 2004 \\
\hline coscaroni & 14.1 & 18.4 & 0.22 & 0.36 & & & $\arg$ & Froehlich 2002 \\
\hline cosnipata & 10.5 & 10.5 & & & 1400 & 1030 & pe & $\begin{array}{l}\text { Stark y Sivec 1998; } \\
\text { Stark } 2004\end{array}$ \\
\hline costana & 8.5 & & & & 840 & & $\mathrm{cr}$ & Stark 1998 \\
\hline cotacachi & 15 & 18 & & & 1250 & & ecu & Stark 2001a \\
\hline cotopaxi & 15.5 & & & & 2000 & & ecu & Stark 2001a \\
\hline cruza & 8 & & & & 810 & 160 & ve, guy & Stark 1995; 1999 \\
\hline cuadrada* & 18 & & & & & & ve & Stark 1995 \\
\hline curiosa & 9 & & & & 700 & 470 & $\mathrm{cr}$ & Stark 1998 \\
\hline cusi & 13.5 & & & & 2060 & 2030 & bo & Stark 2004 \\
\hline cuzco & 18 & & & & 2150 & 1400 & bo & $\begin{array}{l}\text { Stark y Sivec 1998; Stark } \\
2004\end{array}$ \\
\hline digitata & 11.5 & 14.5 & 0.16 & 0.3 & & & ve & Stark 1995 \\
\hline divisa & 15 & 19 & & & 1450 & 250 & $\mathrm{cr}$ & Stark 1998 \\
\hline exquisita & 14 & & & & 1650 & & cr & Stark 1998 \\
\hline
\end{tabular}




\section{APÉNDICE (Continuación)}

\section{Resumen de los datos encontrados}

Códigos: (*) Especie no utilizada por falta de información; (arg) Argentina; (bo) Bolivia; (bra) Brazil; (col) Colombia; (cr) Costa Rica; (ecu) Ecuador; (guy) Guyana; (ho) Honduras; (mex) México; (nic) Nicaragua; (pa) Panamá; (per) Perú; (sur) Suriname; (ve) Venezuela.

\begin{tabular}{|c|c|c|c|c|c|c|c|c|}
\hline \multirow[t]{2}{*}{ Anacroneuria sp. } & \multicolumn{2}{|c|}{$\begin{array}{l}\text { Tamaño del cuerpo } \\
(\mathrm{mm})\end{array}$} & \multicolumn{2}{|c|}{$\begin{array}{c}\text { Huevo } \\
(\mathrm{mm})\end{array}$} & \multicolumn{2}{|c|}{$\begin{array}{l}\text { Altura } \\
(\mathrm{m})\end{array}$} & \multirow[t]{2}{*}{ País } & \multirow[t]{2}{*}{ Bibliografía } \\
\hline & Macho & Hembra & Ancho & Largo & Máxima & Minima & & \\
\hline fenestrata* & 16 & & & & & & ve & Stark 1995 \\
\hline fittkaui & 9.6 & 13.4 & 0.21 & 0.32 & & & bra & Froehlich 2003 \\
\hline flavicoronata & 11 & 11 & & & 380 & & pe & Stark y Sivec 1998 \\
\hline flavifrons & 9 & 11 & & & & & pe & Stark y Sivec 1998 \\
\hline flinti & 10 & & & & 200 & & pe & Stark y Sivec 1998 \\
\hline flintorum* & 11 & & & & & & bra & Froehlich 2002 \\
\hline fuscicosta & 10.1 & 12.23 & & & 700 & & bra & Froehlich 2002 \\
\hline$G U-1^{*}$ & & 11 & & & & & guy & Stark 1999 \\
\hline$G U-2 *$ & & 11 & & & & & guy & Stark 1999 \\
\hline$G U-3 *$ & & 10 & & & & & guy & Stark 1999 \\
\hline$G U-4^{*}$ & & 13 & & & & & guy & Stark 2000 \\
\hline$G U-5^{*}$ & & 13.5 & & & & & guy & Stark 2000 \\
\hline guambiana* & & 30 & & & 2900 & 1400 & col & Stark y Zuniga 2003 \\
\hline guayaquil* $^{*}$ & & & & & 3500 & & col, ecu & Stark 2001a \\
\hline hacha & 11 & & & & 550 & & $\mathrm{cr}$ & Stark 1998 \\
\hline handlirschi & 14 & 16 & & & 1650 & 250 & pe, bo & Stark y Sivec 1998 \\
\hline harperi & 7 & 9 & & & & & pa & Stark 1998 \\
\hline heppneri & 10 & & & & 672 & & pe & Stark y Sivec 1998 \\
\hline hieroglyphica* & & & & & & & ecu & Stark 2001a \\
\hline holzenthali & 12 & 15 & & & 980 & 470 & $\mathrm{cr}$ & Stark 1998 \\
\hline huayna* & 7.5 & & & & & & pe & Stark 2004 \\
\hline impensa & 16.5 & 21 & 0.38 & 0.28 & & & bra, arg & Froehlich 2002 \\
\hline inca & 10 & 12 & & & 1400 & 200 & pe & $\begin{array}{l}\text { Stark y Sivec 1998; Stark } \\
2004\end{array}$ \\
\hline intermixta* & & 29 & & & & & ve & Stark 1995 \\
\hline iridescens $*$ & & 16 & & & & & bo & Stark y Sivec 1998 \\
\hline isleta & 8 & 12 & 0.16 & 0.24 & 400 & & Tobago islar & Stark 1994 \\
\hline jewetti & 14 & & & & 2600 & 1080 & ecu & Stark 2001a \\
\hline jivaro & 18 & & & & 3010 & 2910 & ecu & Stark 2001a \\
\hline karina & 11 & & & & 810 & & ve & Stark 1999 \\
\hline kitchensi & 9 & 13 & & & 900 & & ecu & Stark 2001a; 2004 \\
\hline
\end{tabular}




\section{APÉNDICE (Continuación)}

\section{Resumen de los datos encontrados}

Códigos: (*) Especie no utilizada por falta de información; (arg) Argentina; (bo) Bolivia; (bra) Brazil; (col) Colombia; (cr) Costa Rica; (ecu) Ecuador; (guy) Guyana; (ho) Honduras; (mex) México; (nic) Nicaragua; (pa) Panamá; (per) Perú; (sur) Suriname; (ve) Venezuela.

\begin{tabular}{|c|c|c|c|c|c|c|c|c|}
\hline \multirow[t]{2}{*}{ Anacroneuria sp. } & \multicolumn{2}{|c|}{$\begin{array}{l}\text { Tamaño del cuerpo } \\
(\mathrm{mm})\end{array}$} & \multicolumn{2}{|c|}{$\begin{array}{l}\text { Huevo } \\
(\mathrm{mm})\end{array}$} & \multicolumn{2}{|c|}{$\begin{array}{l}\text { Altura } \\
(\mathrm{m})\end{array}$} & \multirow[t]{2}{*}{ País } & \multirow[t]{2}{*}{ Bibliografía } \\
\hline & Macho & Hembra & Ancho & Largo & Máxima & Minima & & \\
\hline kondratieffi & 11 & & & & 250 & & ecu & Stark 2001a \\
\hline lineata & 12 & & & & 810 & 470 & cr & Stark 1998 \\
\hline llana & 10.5 & 14 & & & 1350 & & guy, ve & Stark 1995; 1999 \\
\hline loreto* & & 9 & & & & & pe & Stark et al. 2001 \\
\hline lupaca & 9 & & & & 250 & & pe & Stark y Sivec 1998 \\
\hline magnirufa & 20 & 26 & & & 1450 & 390 & cr, pa, ho & Stark 1998 \\
\hline major & 23 & & & & 3010 & & ecu & Stark 2001a \\
\hline makushi* & 9.5 & & & & & & guy & Stark 1999 \\
\hline manauensis & 7.1 & 8.7 & & & & & bra & Ribeiro y Froehlich 2001 \\
\hline marca & 16 & 20 & & & 1650 & 480 & cr & Stark 1998 \\
\hline marginata & 8 & & & & 600 & 550 & cr & Stark 1998 \\
\hline maritza & 13 & 16 & & & 470 & 75 & cr & Stark 1998 \\
\hline marlieri & 8.3 & 12 & & & & & bra & Ribeiro y Froehlich 2001 \\
\hline menuda* & 8 & & & & & & ve & Stark 1995 \\
\hline minuta* & 9 & & & & & & bra & Froehlich 2002 \\
\hline minuta & 8.3 & 10.5 & & & & & bra & Ribeiro y Froehlich 2001 \\
\hline moche* & 9 & & & & & & pe & Stark y Sivec 1998 \\
\hline mochica & 9 & 13 & & & 250 & & pe & $\begin{array}{l}\text { Stark y Sivec 1998; Stark } \\
2004\end{array}$ \\
\hline montera & 10 & 13 & & & & & col, pe & $\begin{array}{l}\text { Stark y Sivec 1998; Stark et } \\
\text { al. } 2001\end{array}$ \\
\hline тиеsса & 16 & & & & 1785 & & ve & $\begin{array}{l}\text { Maldonado et al. 2002; } \\
\text { Stark } 1995\end{array}$ \\
\hline nazca & 20 & & & & 2400 & 1600 & pe & Stark y Sivec 1998 \\
\hline novateutonia & 11.1 & 13.7 & 0.22 & 0.39 & & & bra, arg & Froehlich 2002 \\
\hline oculatila & 8.3 & 12.7 & 0.17 & 0.3 & & & bra, arg & Froehlich 2002 \\
\hline ohausiana* & 20 & & & & & & ecu & Stark 2001a \\
\hline pacaje & 14 & & & & 1600 & 1400 & bo & Stark y Sivec 1998 \\
\hline pachacuti & 11 & & & & 2400 & 1100 & pe, bo & Stark y Sivec 1998 \\
\hline pakitza & 9.5 & 13.5 & & & 900 & 250 & pe & Stark y Sivec 1998 \\
\hline paleta & 15 & & & & 1650 & 872 & ve & $\begin{array}{l}\text { Maldonado et al. 2002; } \\
\text { Stark 1995; } 1999\end{array}$ \\
\hline
\end{tabular}




\section{APÉNDICE (Continuación)}

\section{Resumen de los datos encontrados}

Códigos: (*) Especie no utilizada por falta de información; (arg) Argentina; (bo) Bolivia; (bra) Brazil; (col) Colombia; (cr) Costa Rica; (ecu) Ecuador; (guy) Guyana; (ho) Honduras; (mex) México; (nic) Nicaragua; (pa) Panamá; (per) Perú; (sur) Suriname; (ve) Venezuela.

\begin{tabular}{|c|c|c|c|c|c|c|c|c|}
\hline \multirow[t]{2}{*}{ Anacroneuria sp. } & \multicolumn{2}{|c|}{$\begin{array}{l}\text { Tamaño del cuerpo } \\
(\mathrm{mm})\end{array}$} & \multicolumn{2}{|c|}{$\begin{array}{l}\text { Huevo } \\
(\mathrm{mm})\end{array}$} & \multicolumn{2}{|c|}{$\begin{array}{l}\text { Altura } \\
(\mathrm{m})\end{array}$} & \multirow[t]{2}{*}{ País } & \multirow[t]{2}{*}{ Bibliografía } \\
\hline & Macho & Hembra & Ancho & Largo & Máxima & Minima & & \\
\hline paria & 10 & 12 & & & 810 & 15 & ve & Stark 1999 \\
\hline parva* & 8.5 & & & & & & ecu & Stark 2001a \\
\hline pastaza & 8 & 10 & & & & & ecu & Stark 2001a \\
\hline$P E-1 *$ & & 10 & & & & & pe & Stark y Sivec 1998 \\
\hline pellucida* & & 16 & & & & & pe & Stark y Sivec 1998 \\
\hline реquena & 8.5 & 11.5 & 0.19 & 0.3 & 140 & & ve & Stark 1995 \\
\hline perija & 9 & & & & 360 & & ve & Stark 1999 \\
\hline perplexa & 8 & & & & 1150 & 30 & cr & Stark 1998 \\
\hline perpusilla & 8 & & & & 250 & & pe & Stark y Sivec 1998 \\
\hline petersi & 9.2 & 11.4 & & & & & bra & Froehlich 2002 \\
\hline phantoma & 10 & & & & 1350 & & guy & Stark 1999 \\
\hline pictipes & 10 & 11 & & & 2350 & & guy & Stark 1999 \\
\hline pinza & 10 & 12 & 0.29 & 0.4 & 760 & 140 & ve & Stark 1995 \\
\hline pistacina* & & & & & & & ecu & Stark 2001a \\
\hline planada* & & 17 & & & 1920 & 503 & col, ecu & Stark 2001a \\
\hline planicollis & 15 & 17 & & & 1400 & & $\begin{array}{l}\text { cr, pa, mex, } \\
\text { nic, puntarenas }\end{array}$ & S Stark 1998 \\
\hline plaumanni & 13.8 & 17.1 & 0.2 & 0.42 & & & bra, arg & Froehlich 2002 \\
\hline plutonis & 21 & 28 & & & 1990 & 550 & $\mathrm{cr}$, trinidad & Stark 1998 \\
\hline portilla* & & & & & 1080 & & col, ecu & Stark 2001a \\
\hline pucallpa* & 10 & & & & & & pe & Stark 2004 \\
\hline puna & 11 & & & & 2000 & & ecu & Stark 2001a \\
\hline quechua & 14 & & & & 1700 & 1600 & pe & Stark y Sivec 1998 \\
\hline quijo & 7.5 & 8.5 & & & & & ecu & Stark 2001a \\
\hline quilla* & & & & & 3010 & 2000 & col, ecu & Stark 2001a \\
\hline rawlinsi & 17.5 & & & & 2200 & & ecu & Stark 2001a \\
\hline rondoniae & 9.7 & 13.2 & & & & & bra & Froehlich 2002 \\
\hline rosita* & & & & & & & col, ecu & Stark 2001a \\
\hline rossi* & & 10 & & & & & pe & Stark 2004 \\
\hline rugosa* & 9.5 & & & & & & ecu & Stark 2001a \\
\hline saltensis & 15.5 & 18.2 & 0.31 & 0.18 & & & $\arg$ & Froehlich 2002 \\
\hline
\end{tabular}




\section{APÉNDICE (Continuación)}

\section{Resumen de los datos encontrados}

Códigos: (*) Especie no utilizada por falta de información; (arg) Argentina; (bo) Bolivia; (bra) Brazil; (col) Colombia; (cr) Costa Rica; (ecu) Ecuador; (guy) Guyana; (ho) Honduras; (mex) México; (nic) Nicaragua; (pa) Panamá; (per) Perú; (sur) Suriname; (ve) Venezuela.

\begin{tabular}{|c|c|c|c|c|c|c|c|c|}
\hline \multirow[t]{2}{*}{ Anacroneuria sp. } & \multicolumn{2}{|c|}{$\begin{array}{l}\text { Tamaño del cuerpo } \\
(\mathrm{mm})\end{array}$} & \multicolumn{2}{|c|}{$\begin{array}{l}\text { Huevo } \\
(\mathrm{mm})\end{array}$} & \multicolumn{2}{|c|}{$\begin{array}{l}\text { Altura } \\
(\mathrm{m})\end{array}$} & \multirow[t]{2}{*}{ País } & \multirow[t]{2}{*}{ Bibliografía } \\
\hline & Macho & Hembra & Ancho & Largo & Máxima & Minima & & \\
\hline schmidti* & & 10.5 & & & & & ecu & Stark 2001a \\
\hline segnini & 12 & 14 & & & 1650 & 1260 & ve & Maldonado et al. 2002 \\
\hline shamatari & 19 & 24 & 0.22 & 0.45 & 2100 & 760 & ve & Stark 1995 \\
\hline signata* & & 13 & & & & & ve & Stark 1995 \\
\hline socapa* & & 26 & & & 2200 & 1460 & co & Stark y Zuñiga 2003 \\
\hline $\operatorname{sp} . A^{*}$ & & & & & 2200 & & ve & Maldonado et al. 2002 \\
\hline spanleri & 9.5 & 12 & & & & & ecu & Stark 2001a \\
\hline spectori & 16 & & & & 2060 & 2030 & bo & Stark 2004 \\
\hline stanjewetti & 9.5 & 13.5 & 0.19 & 0.42 & & & bra, arg & Froehlich 2002 \\
\hline subcostalis & & 14.2 & 0.21 & 0.37 & & & bra & Froehlich 2002 \\
\hline tachira & 24 & 30 & & & 2300 & & ve & Stark y Zuñiga 2003 \\
\hline takutu & 8.5 & 10.5 & 0.21 & 0.26 & & & guy & Stark 2000 \\
\hline talamanca & 8 & 10 & & & 200 & 30 & $\mathrm{cr}, \mathrm{pa}$ & Stark 1998 \\
\hline taylori & 10.5 & 14 & & & 400 & 250 & pe & Stark 2004 \\
\hline tena & 9 & & & & 520 & & ecu & Stark 2001a \\
\hline timote & 17 & & & & 2400 & & ve & Stark 1999 \\
\hline tinctilamella & & 10.9 & 0.23 & 0.39 & & & bra, arg & Froehlich 2002 \\
\hline tiwanaku* & & 11.5 & & & 1600 & & bo & Stark 2004 \\
\hline toriba* & 10.6 & & & & & & bra & Froehlich 2002 \\
\hline tornada & 16 & & & & 1400 & & $\mathrm{cr}$ & Stark 1998 \\
\hline trimacula & 12.2 & 17.1 & 0.25 & 0.36 & & & bra, arg & Froehlich 2002 \\
\hline tungurahua* & 15 & & & & & & ecu & Stark 2001a \\
\hline tzapino & 8 & & & & 400 & & ecu & Stark 2001a \\
\hline uatsi & 9 & 10 & & & 90 & 15 & $\mathrm{cr}$ & Stark 1998 \\
\hline ucumari & 26 & & & & 2400 & 2000 & ve & Stark y Zuñiga 2003 \\
\hline uru & 21 & & & & 2500 & 900 & pe, bo & Stark y Sivec 1998 \\
\hline uyara & 14.2 & 19.7 & 0.28 & 0.36 & & & bra, arg & Froehlich 2002 \\
\hline varilla & 10 & 13 & & & 700 & 550 & cr, pa & Stark 1998 \\
\hline ventana & 15 & & & & 1650 & 1400 & $\mathrm{cr}$ & Stark 1998 \\
\hline vilcabamba & 17 & & & & 2400 & 16 & pe & Stark y Sivec 1998 \\
\hline vistosa & 9.5 & 13.5 & 0.19 & 0.25 & 140 & & ve & Stark 1995 \\
\hline
\end{tabular}




\section{APÉNDICE (Continuación)}

\section{Resumen de los datos encontrados}

Códigos: (*) Especie no utilizada por falta de información; (arg) Argentina; (bo) Bolivia; (bra) Brazil; (col) Colombia; (cr) Costa Rica; (ecu) Ecuador; (guy) Guyana; (ho) Honduras; (mex) México; (nic) Nicaragua; (pa) Panamá; (per) Perú; (sur) Suriname; (ve) Venezuela.

\begin{tabular}{|c|c|c|c|c|c|c|c|c|}
\hline \multirow[t]{2}{*}{ Anacroneuria sp. } & \multicolumn{2}{|c|}{$\begin{array}{c}\text { Tamaño del cuerpo } \\
(\mathrm{mm})\end{array}$} & \multicolumn{2}{|c|}{$\begin{array}{l}\text { Huevo } \\
(\mathrm{mm})\end{array}$} & \multicolumn{2}{|c|}{$\begin{array}{l}\text { Altura } \\
(\mathrm{m})\end{array}$} & \multirow[t]{2}{*}{ País } & \multirow[t]{2}{*}{ Bibliografía } \\
\hline & Macho & Hembra & Ancho & Largo & Máxima & Minima & & \\
\hline vitripennis* & 12 & & & & & & pe & Stark y Sivec 1998 \\
\hline$V Z-1$ & & 20 & 0.2 & 0.33 & & & ve & Stark 1995 \\
\hline$V Z-10^{*}$ & & & & & 1450 & & ve & Stark 1995 \\
\hline$V Z-11 *$ & & 17 & & & 2500 & & ve & Maldonado et al. 2002 \\
\hline$V Z-12 *$ & & 17 & & & 872 & & ve & Maldonado et al. 2002 \\
\hline$V Z-2$ & & 18 & 0.2 & 0.38 & 2500 & & ve & Stark 1995 \\
\hline$V Z-3 *$ & & 16.5 & & & & & ve & Stark 1995 \\
\hline$V Z-4 *$ & & 13 & & & & & ve & Stark 1995 \\
\hline$V Z-5^{*}$ & & 13.5 & & & & & ve & Stark 1995 \\
\hline VZ-6* & & 13 & & & & & ve & Stark 1995 \\
\hline$V Z-7 *$ & & 10 & & & & & ve & Stark 1995 \\
\hline$V Z-8^{*}$ & & 11 & & & & & ve & Stark 1995 \\
\hline$V Z-9$ & & 11 & 0.16 & 0.31 & & & ve & Stark 1995 \\
\hline wapishana* & & 11 & & & 1350 & & guy & Stark 1999 \\
\hline wincha & 10 & & & & 1700 & 1400 & pe & Stark y Sivec 1998 \\
\hline woytkowskii & 8.5 & & & & 900 & & pe & Stark y Sivec 1998 \\
\hline xinguensis & 9.5 & 11.2 & 0.21 & 0.35 & & & bra & Froehlich 2002 \\
\hline$x$-nigrum $*$ & 9 & & & & & & pe & Stark y Sivec 1998 \\
\hline уатео & 10 & & & & 450 & 120 & pe, bo & Stark y Sivec 1998 \\
\hline ytuguazu* & 9.1 & & & & & & $\arg$ & Froehlich 2002 \\
\hline zamora & 13 & & & & 1340 & & ecu & Stark 2001a \\
\hline zapata & 14 & & & & 1400 & 600 & $\mathrm{cr}$ & Stark 1998 \\
\hline zarpa* & 10 & & & & & & pa & Stark 1998 \\
\hline zwicki & 13 & & & & 1700 & 900 & pe, bo & Stark y Sivec 1998 \\
\hline
\end{tabular}


